\title{
3D Modeling of Dendritic Grain Structures in Turbine Blade Investment Cast Parts
}

\author{
Ch.-A. Gandin(a), M. Rappaz(a), J.-L. Desbiolles(a), \\ E. Lopez $(b)$, M. Swierkosz ${ }^{(b)}$, and Ph. Thévoz $(b)$ \\ (a) Ecole Polytechnique Fédérale de Lausanne \\ Laboratoire de métallurgie physique \\ CH-1015 Lausanne, Switzerland \\ (b) CALCOM S.A., PSE-Ecublens \\ CH-1015 Lausanne, Switzerland
}

\begin{abstract}
A three-dimensional (3D) Cellular Automaton - Finite Element (CAFE) model is presented for the modeling of dendritic solidification grain structures. It is applied to the prediction of the grain structure formed in investment cast parts, demonstrating its ability to reproduce the growth competition occurring among columnar grains in a pig-tail grain selector, the extension of a grain in an undercooled region of liquid (re-entrant corner), as well as polycrystalline growth in Directionally Solidified (DS) turbine blades. This 3D CAFE model is a very valuable metallography tool for the design of investment cast parts. In particular, it allows to study grain defects such as spurious nucleation of stray crystals in undercooled regions, grain selection and grain texture evolutions.
\end{abstract}

\footnotetext{
Superalloys $718,625,706$ and Various Derivatives

Edited by E.A. Loria

The Minerals, Metals \& Materials Society, 1997
} 


\section{Introduction}

The investment casting process is routinely used to produce components of turbines of modern aircraft engines and land-based power-plants. The mechanical properties of such nickel-base cast parts at high temperatures are tremendously contingent on the solidification grain structures. However, the control of grain structures remains a difficult task for casting designers and foundrymen. In particular, severe obstacles are encountered to cast defect-free single crystal turbine blades.

In order to increase the yield rate of the investment casting process, several modeling approaches have been carried out. They can be divided into two categories. The first type of models are related to the prediction of grain defects on the basis of the process parameters, mainly the thermal gradient, $G$, and the velocity of the isotherms, v. Results are depicted by curves or windows in G-v diagrams representing the limits between reliable and inappropriate casting conditions. These limits are predicted using analytical calculations to model the relevant physical mechanisms. Examples are found for the prediction of freckles, porosity formation [1-3] and spurious grain nucleation [4-6] during directional solidification of superalloys.

For the modeling of grain defects, de Bussac and Gandin [6] have recently shown that several limitations are met with this type of models due to the numerous assumptions of the analytical approach. For instance, an exact description of the geometry of the mold, the crystallographic orientation of the grains and the precise cooling conditions of the whole part can only be approximated by such models. In most cases, it is necessary to rely on numerical approaches which are the second class of models. However, in this category, most of the work has focused on a macroscopic approach (heat flow, radiation, convection, etc.) and very few models are capable of coupling such aspects with a metallurgy-based microscopic approach.

In this contribution, a three-dimensional (3D) numerical model for the prediction of grain structures in investment cast parts is presented. It combines Finite Element (FE) heat flow computations at the macroscopic scale with a Cellular Automaton (CA) description of nucleation and growth of dendritic grains. It will be applied to the directional solidification of a pig-tail shape geometry (grain selector) used for the production of single crystal turbine blades.

\section{Three-Dimensional Cellular Automaton - Finite Element Model}

The 3D CAFE model is an extension of the two-dimensional model already published in the literature [7-8]. However, due to the very large number of cells defined in 3D (a few 10 millions), the memory limitations of current workstations require to use specific numerical techniques which are briefly explained below together with the CAFE algorithm.

\section{Grid generation and Initialization}

Because cells are numerous, they are defined in a dynamic way only in the zones of interest, i.e., as soon as a region has a temperature below the liquidus temperature, $\mathrm{T}_{\mathrm{L}}$. For that purpose, only blocks are defined at the beginning of the simulation (see Figure 1). The block numbering is uniquely defined within a parallelepiped (window) for which the CA calculation will be performed. The limits of this window are chosen by the user to assess the grain structure formation in a critical area or in the whole casting. The blocks falling totally or partially within the casting are "asleep" at the beginning of the simulation, whereas those located outside are "dead". When a block sees the temperature of one nodal point of the finite element to which it is connected fall below $\mathrm{T}_{\mathrm{L}}$, then it becomes an active block: cells are defined within this block to perform the CA calculation. In the case of Figure 1, the blocks are typically made of $6 \times 6 \times 6$ cells (in $3 D$ ). 


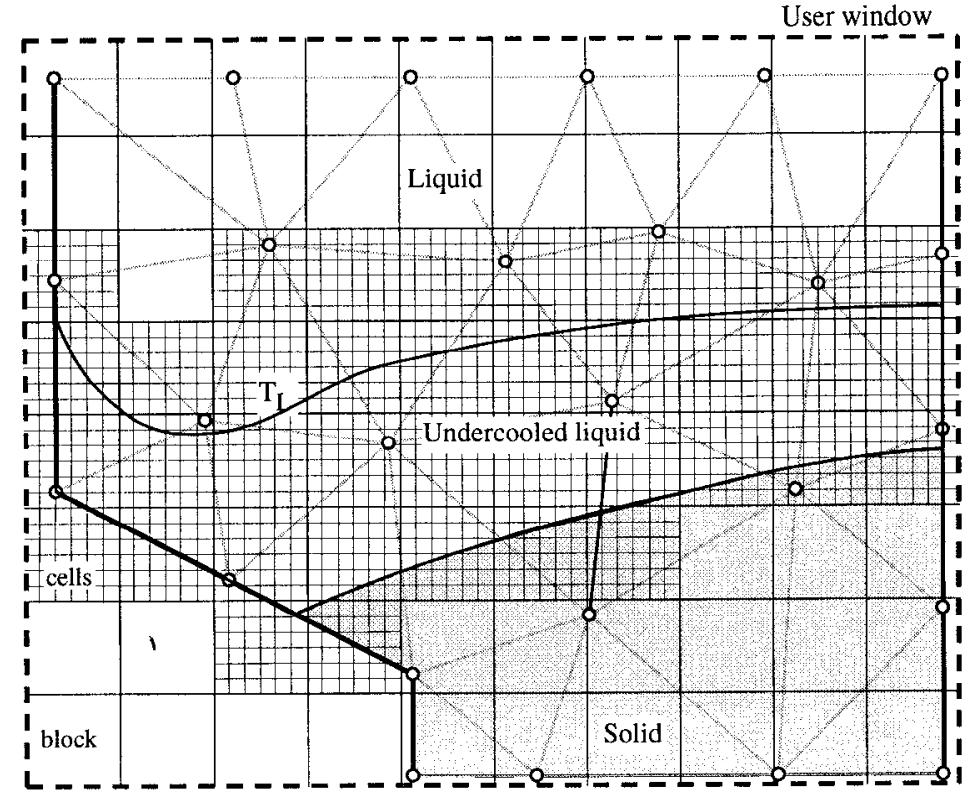

Figure 1 Schematics of the Cellular Automaton - Finite Element calculation showing the intermediate block structure and the zone where cells are dynamically defined.

1- Cell numbering. Each cell is defined within a block by its number, which varies from 1 to 216 for the $6 \times 6 \times 6$ cell blocks shown in Figure 1. The cell numbering is therefore not unique within the user window. However, the position of a cell is uniquely defined and can thus be calculated when its number is combined with its block number. The same remark applies for the cell neighborhood. The finite element number to which the cell $v$ belongs is stored in the index $E_{V}$. This index is used for temperature interpolation from the FE nodes of element $E_{V}$ to the cell $v$.

2- Cell reference initialization. For each cell standing at the limit of the casting and, if specified, at the limit of the user window, a reference index, $R_{V}$, is assigned. It corresponds to the reference of a FE boundary or to a reference imposed on one face of the user window. This index is used for imposing nucleation boundary conditions.

3- Cell state initialization. At the beginning of the calculation, all the cells are liquid and have a state index, $I_{V}$, equal to zero. During the calculation, this state index will be switched to an integer value when it becomes mushy, as a result of a nucleation event or growth from neighboring mushy cells.

As soon as all the state indices of the cells of a block are non-zero, the block is "killed" in order to release the memory space associated with these cells. Only the state index of the block is kept. In such a way, the memory used is optimized so as to track the growth of the solid - liquid interface and the undercooled region of liquid where nucleation is likely to proceed. In figure 1, the zone where blocks are active is easily visualized by the finest grid region representing the $\mathrm{CA}$ cells.

Figure 2 illustrates the grid generation for a close-up detail of a turbine blade geometry (junction between a pig-tail selector and the blade platform). The typical cell size used in the CA calculation is $100 \mu \mathrm{m}$. Since the cell grid is automatically defined from the FE mesh with all the boundary reference and element indices, no additional work is required once the FE mesh is given. 


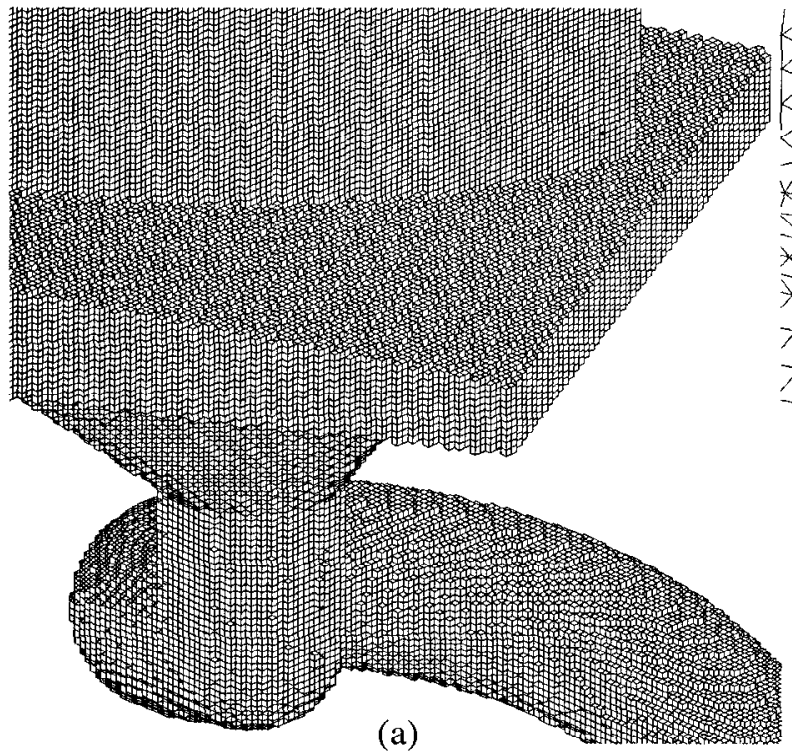

(a)

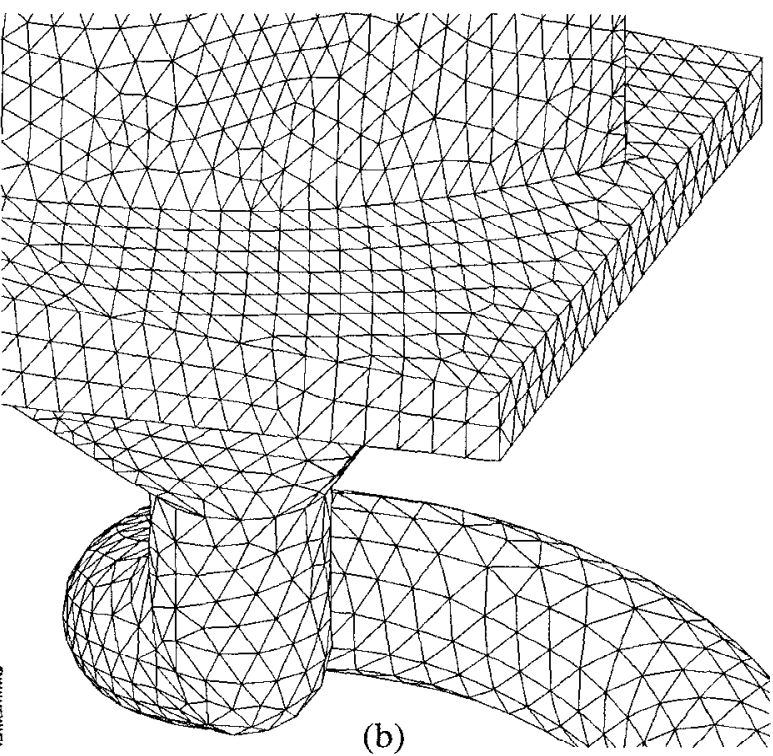

(b)

Figure 2 (a) Cellular Automaton grid automatically generated from (b) the Finite Element mesh showing a detail of an investment cast part geometry.

\section{Nucleation and Growth Algorithms}

When first activated, a block is composed of surface cells (i.e., referenced cells with $R_{V} \neq 0$ ), volume cells (i.e., unreferenced cells with $R_{V}=0$ ) and possibly cells falling outside the casting. The number of nucleation sites within the block can be obtained by multiplying the total volume of unreferenced cells by the volumetric nucleation density. The same calculation is performed for referenced cells, multiplying the surface nucleation density by the area of the referenced cells projected on the corresponding face of the FE mesh. Potent nucleation cells, $v$, and critical undercooling, $\Delta \mathrm{T}$ nucl, are then randomly attributed to the cells of the block according to gaussian distributions of nucleation sites, $\mathrm{n}_{\mathrm{v}}(\mathrm{s})(\Delta \mathrm{T})$, where $(\mathrm{v})$ and $(\mathrm{s})$ correspond to bulk and surface nucleation, respectively $[7,8]$. The procedure is similar to that used in the 2D CAFE model and requires to define for each nucleation distribution ( $\mathrm{v}$ or $\mathrm{s}$ ) the mean undercooling, $\Delta \mathrm{T}_{\mathrm{N}}$, the standard deviation, $\Delta \mathrm{T}_{\sigma}$, and the maximum nucleation density, $\mathrm{n}_{\max }$. Once a new grain has formed at a cell location, the index of this cell is switched to a randomly selected integer representing its orientation (three Euler angles) with respect to the CA axes. It then grows with a dendritic morphology.

The 3D CA growth algorithm has been recently published. It is based on a decentred octahedron algorithm whose details can be found in Ref. 9. It is just reminded here that the octahedron has $<100>$ diagonals and $\{111\}$ faces: in a uniform temperature field, it is assumed that a single grain grows from a cell center with such a morphology. The size of this octahedron is given by the integration of the growth kinetics of the dendrite tips, $v(\Delta T)$, where $\Delta \mathrm{T}$ is the undercooling. In a non-uniform temperature field, small octahedra are grown from all the cells which are mushy. At some time, these small octahedra will "capture" neighboring cells which are still liquid. These cells in turn will have their state index changed to the state index of the parent nucleus. This allows to propagate the dendritic network from cells to cells, while using the local undercooling of each cell. In order not to bias the original misorientation of the grain by the CA axes, it is necessary to introduce a virtual growth center for each cell, which is not necessarily identical to the cell center. More details can be found in Ref. 9. It should be pointed out that a 26-cells neighborhood (i.e., first, second and third neighbors in a cubic lattice) has been used in the present calculations. 
Figure 3 shows the calculated shape of a single grain growing in a Bridgman-type configuration (i.e., constant thermal gradient and cooling rate). The gradient is along the $\mathrm{Z}$-direction and the $<100>$ axes of the grain are rotated with respect to the $\mathrm{X}_{-}, \mathrm{Y}_{-}$, and $\mathrm{Z}$-axes. Figure $3 \mathrm{a}$ corresponds to an analytical solution [9] whereas Figure $3 \mathrm{~b}$ is the result of the CA growth algorithm. As can be seen, the preferred $<100>$ crystallographic orientations of the grain (corresponding to the dendrite trunks and arms directions), as well as the extension of the grain and its envelope are well reproduced by the CA growth algorithm. Under non-uniform temperature conditions, it can be seen that the overall envelope of the grain is no longer that of a regular octahedron and that faces located downstream of the heat flow grow faster than upstream faces.
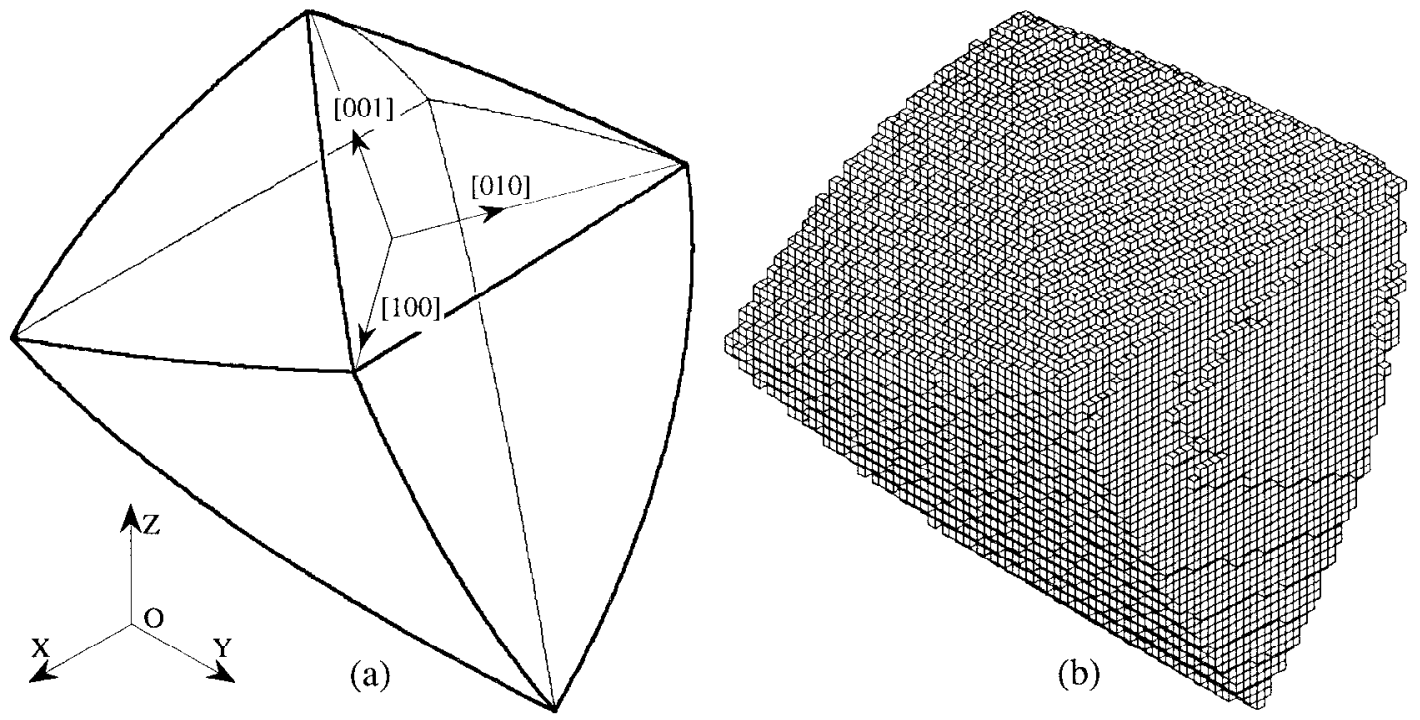

Figure 3 Analytical (a) and CA (b) predictions of the extension of a single crystal in an imposed Bridgman-type temperature field (gradient, $\mathrm{G}=250 \mathrm{~K} / \mathrm{m}$, and cooling rate, $\dot{\mathrm{T}}=-0.1 \mathrm{~K} / \mathrm{s}$ ). Nucleation undercooling, $2 \mathrm{~K}$, growth kinetics relationship, $\mathrm{v}=\mathrm{A} \cdot \Delta \mathrm{T}^{2}$ where $A=10^{-4} \mathrm{~m} /\left(\mathrm{sK}^{2}\right)$, cell size, $l=100 \mu \mathrm{m}$ and Euler angles defining the $<100>$ directions with respect to the X-, Y-, Z-axes $\left(\phi_{1}, \phi, \phi_{2}\right)=\left(20^{\circ}, 20^{\circ}, 20^{\circ}\right)$.

\section{Coupling with FE Calculations and Flow Chart}

At present, the 3D CAFE model is semi-coupled: from the temperature field at each time step, the temperatures are interpolated at the cell locations in order to perform the CA calculations. However, there is no feedback of the latent heat released by the cells: latent heat is taken into account at the macro level using a unique solidification path. It should be pointed out that, as for the micro-enthalpy method of Thévoz et al [10], two time steps are used. The macro time step, $\Delta \mathrm{t}_{\text {macro }}$, is used for the heat flow computations, whereas the growth algorithm uses a micro time step, $\delta t_{\text {micro }}$. This step is such that

$$
\delta \mathrm{t}_{\text {micro }}=\frac{l}{\mathrm{v}\left(\Delta \mathrm{T}_{\max }\right)}
$$

where $l$ is the cell spacing and $\mathrm{v}\left(\Delta \mathrm{T}_{\max }\right)$ is the maximum growth velocity of the dendritic network calculated using a multicomponent dendrite growth kinetics model [11-12]. 


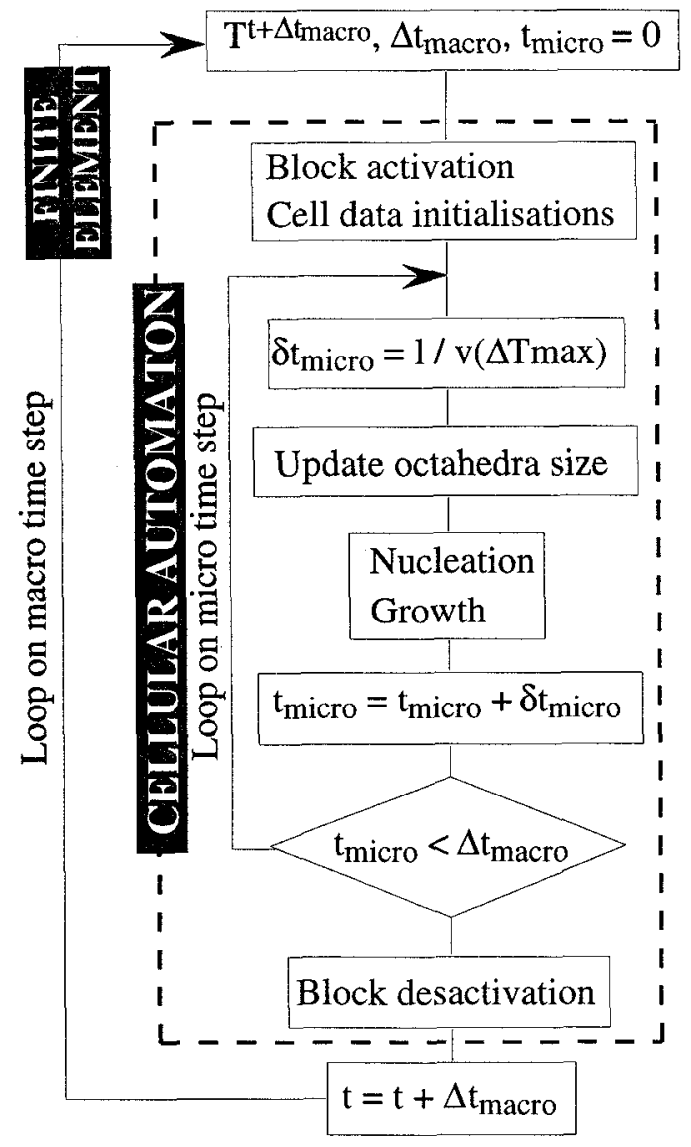

Figure 4 Flow chart of the 3D Cellular Automaton - Finite Element model.

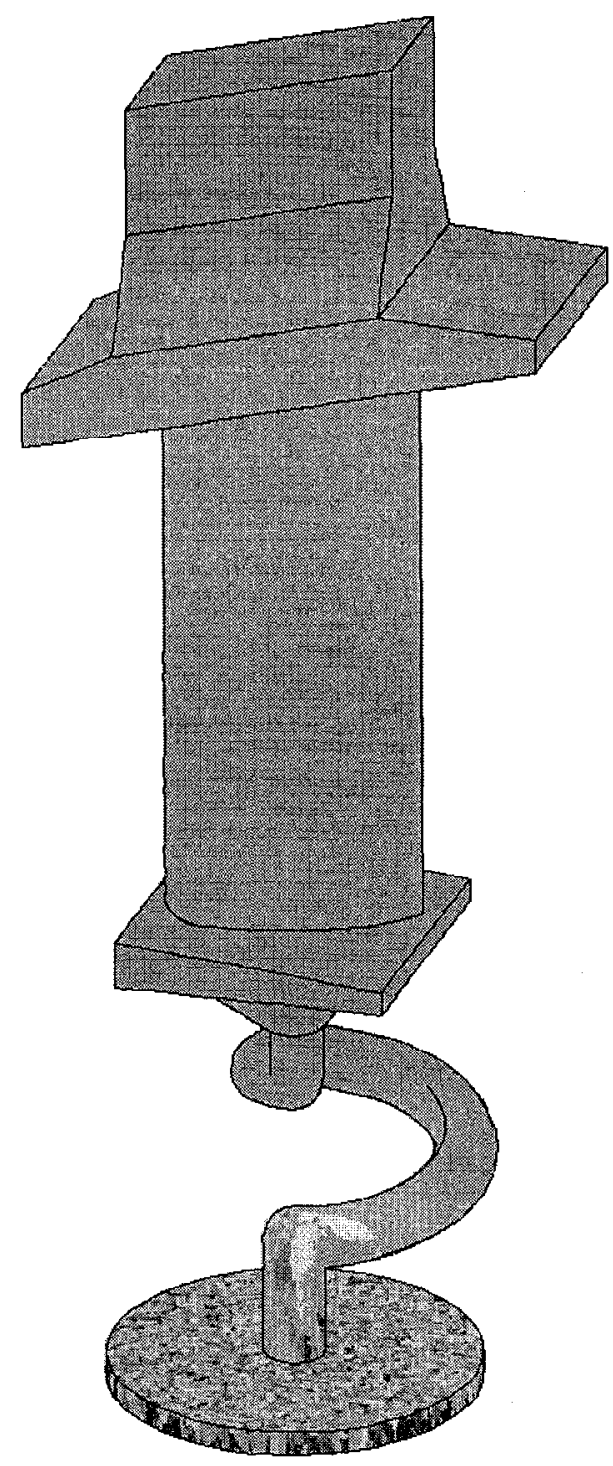

Figure 5 Predicted dendritic grain structure in a turbine blade investment cast part. Cell size, $l=200 \mu \mathrm{m}, \mathrm{CPU}$ time on a Silicon Graphics R4400@250MHz: 7 h. 57 min., number of cells in the casting: $1 ' 863^{\prime} 618,5000$ time steps.

In the semi-coupled scheme, the CAFE model is described by the flow chart of Figure 4:

At each time step, the temperatures are known from the FE calculation using an implicit enthalpy scheme. A loop is then made on all elements in order to activate the blocks belonging to the elements which are susceptible of becoming mushy (i.e., which have at least one node with a temperature below $\mathrm{T}_{\mathrm{L}}$ ). It also performs all initializations due to the activation of the blocks (e.g., random distribution of nucleation cells within a block). 
A loop on the micro time steps is then made until the macro time step is reached. Within this loop, three loops are made on all active blocks. The first one determines the maximum undercooling of the potent nucleation and growing cells, $\Delta \mathrm{T}_{\max }$. This value is used to determine the micro time step, $\delta t_{\text {micro, }}$ according to Eq. (1). This value can change during a macro time step since new mushy cells appear. The second loop calculates the new size of all octahedra associated with the active cells. The third loop updates the new nucleation cells and determines whether the updated octahedra capture new liquid cells. The growth algorithm is run for all solid-liquid interface cells, i.e., all the cells having a non-zero state index $\left(\mathrm{I}_{\mathrm{V}} \neq 0\right)$ and at least one remaining liquid neighboring cell.

Finally, prior to go back to the next macro time step, a final loop desactivates the blocks which have no longer liquid neighboring cells. The memory associated with the cells indices is then released.

\section{Solidification Modeling of a Single Crystal Investment Cast Part}

Figure 5 presents the grain structure calculated in an investment cast turbine blade using the 3D CAFE model. In this simulation, the nucleation parameters are only defined for the small plate at the bottom part of the casting in contact with a copper chill. A sharp gaussian distribution has been chosen simulating a burst of nucleation just below the liquidus temperature. As can be seen from the various gray levels, many grains form in the bottom plate. Some of them enter the vertical cylinder located below the pig-tail selector. The shape of this part of the casting is such that only one grain survives the competition occurring among the columnar grains. This grain then extends freely in the whole blade without any risk of a spurious grain nucleation since no nucleation laws have been specified for the bulk of the liquid or for the inner surface of the ceramic mold.

Figure 6 shows a close-up of the grain structure in the pig-tail selector as calculated with an increased density of cells. A very high flux leaves the bottom part of the casting which is in contact with a copper chill. The thermal properties of the mold and of the insulation materials are such that the other surfaces of the casting can be considered as nearly adiabatic. Accordingly, the thermal gradient direction follows the axis of the bottom cylinder and of the helix selector. It should be pointed out that the selection of the grains having one of their $<100\rangle$ directions most closely aligned with the heat flow direction is essentially achieved before the helix. This has been confirmed experimentally using an electron backscattered diffraction technique for the determination of the grain texture evolution near to the copper chill [13]. After a few millimeters, all the grains surviving the growth selection have their $<100>$ dendrite trunks within only a few degrees to the thermal gradient direction (Z-axis in Figures 5 and 6). This rapid evolution of the grain texture is also reproduced by the CAFE model, as shown in Figure $6 \mathrm{~b}$. In this figure, the pole figure of the $<100\rangle$ crystallographic orientations have been plotted for all the grains still present at the level of section A in Figure 6a. Only one grain is present in section B (see Figure 6a). It has been selected in the helix part and is identified with small circles in Figure $6 \mathrm{~b}$.

Please note that the projection onto a XY plane of the helix direction starting just above section $\mathrm{A}$ is given by the arrow drawn in Figure 6b. It corresponds to a $45^{\circ}$ rotation with respect to the $\mathrm{Y}$-axis. As a consequence of the growth competition at the beginning of the pig-tail selector, one of the $\langle 100\rangle$ crystallographic directions of the selected grain is aligned with this initial helix direction. This means that the selector has selected one of the grains having a secondary arm best aligned with its initial helix direction. It is interesting to note that this is the only contribution of the helix since the primary direction of the dendrites was already selected due to the growth competition in the bottom cylinder. 


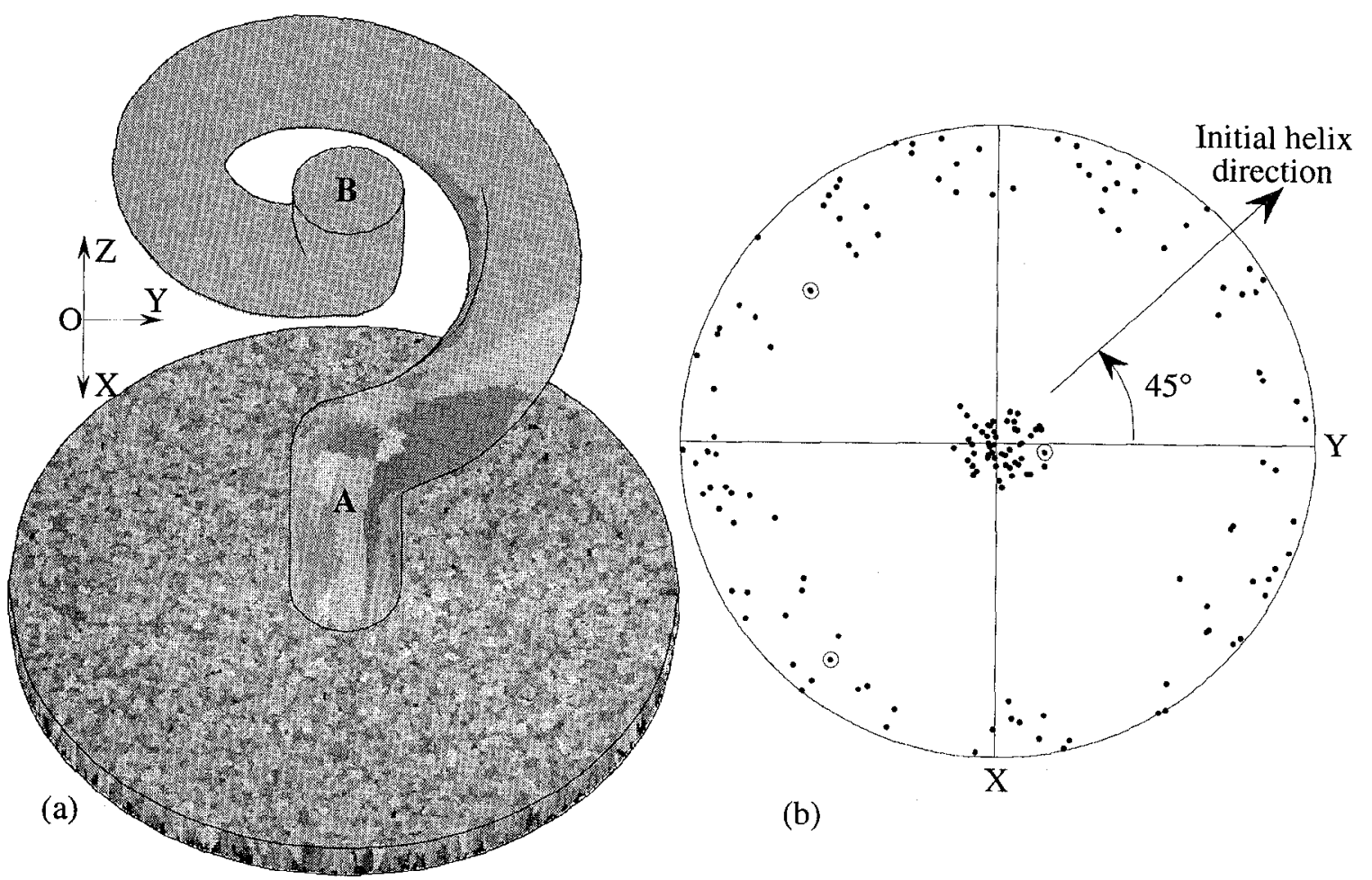

Figure 6 (a) Predicted dendritic grain structures in a pig-tail selector and (b) associated pole figures of the $<100>$ crystallographic orientations of the selected grains at the level of section $\mathrm{A}$. The final grain selected in the helix is identified with small circles. Cell size, $l=100 \mu \mathrm{m}$, nucleation parameters, $\Delta \mathrm{T}_{\mathrm{N}}=0.3 \mathrm{~K}, \Delta \mathrm{T}_{\sigma}=0.3 \mathrm{~K}, \mathrm{n}_{\mathrm{C}}=10^{8} \mathrm{~m}^{-2}$, CPU time on a Silicon Graphic R4400@250MHz: 1 h 17 min., number of cells in the casting: 2'698'391, 1000 time steps.

The model handles the stochastic nature of the crystallographic orientation of the nuclei formed at the bottom surface of the small cylindrical plate of the casting in contact with the copper chill. This is achieved by attributing random values of the Euler angles to the newly formed grains. By changing the initial (seed) value of the algorithm used to generate these random values, the crystallographic orientation of the grains entering the selector can be changed randomly, thus simulating different casting experiments. Therefore, it becomes possible to analyze statistically the crystallographic orientations of the selected grain coming out of the helix gcometry. Figure 7 shows the results obtained when considering ten different calculations. Firstly, as expected, all the primary trunks are well aligned with the normal to the copper chill (i.e., Z-axis at the center of the stereographic projection). The directions are however slightly tilted towards the initial helix direction since the heat flow direction almost follow the geometry of the part if insulated ceramic mold walls are considered. Secondly, eight of the single crystals selected by the pig-tail selector have a secondary arm orientation within \pm 15 degrees of the initial helix direction (see circled points). Only two calculations lead to clearly differently oriented arms (see squared points). This type of numerical experiments could be compared to real ones carried out in a foundry environment, assuming that the cooling conditions are sufficiently well known and reproduced by the FE calculation. 


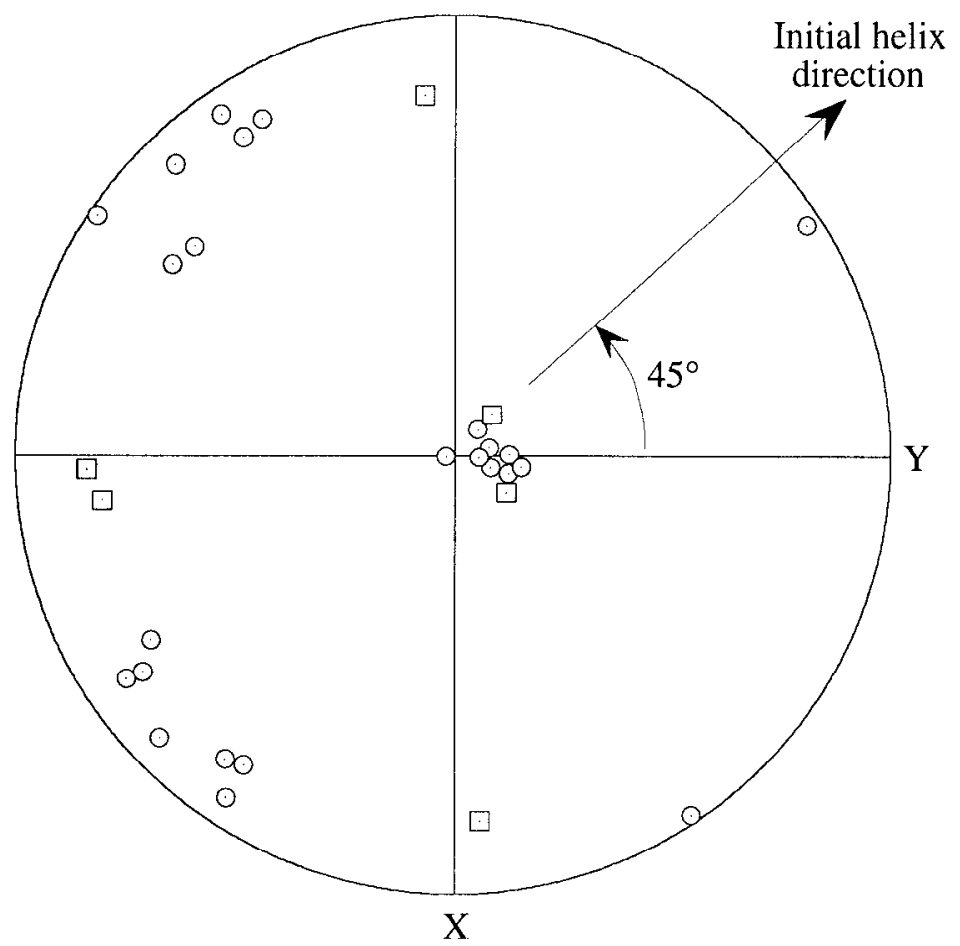

Figure 7

Predicted $<100>$ pole figure of the single crystals selected by the pig-tail selector shown in Figure Ga when ten successive calculations are performed.

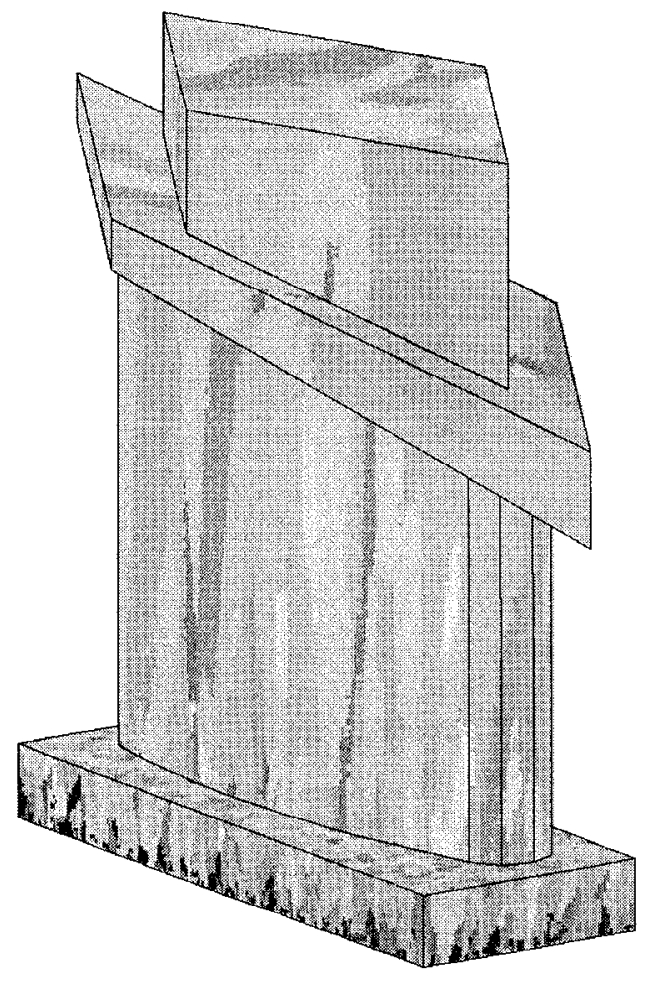

\section{Figure 8}

Predicted columnar grain structure in a DS turbine blade. As the columnar grains grow from the bottom to the top of the blade, their transverse dimension increases as a result of the grain competition. 
Finally, a calculation was performed to illustrate the ability of the model to deal with other casting conditions. Figure 8 shows the predicted columnar grain structure in a DS turbine blade. As can be seen, together with the grain texture evolution observed during directional solidification of dendritic alloys (Figures 6 and 7), the model predicts the characteristic grain density evolution seen in transverse cross sections. The increase of the transverse size of the columnar grains from the bottom to the top of the blade shown in Figure 8 is a result of the suppression of the grains badly aligned with the main blade direction, which also corresponds with the main thermal gradient direction.

\section{Conclusions}

The 3D CAFE model presented in this contribution is shown to reproduce features of solidification grain structures that were never modeled before. In particular, the growth competition occurring among columnar grains are directly reproduced, taking into account the crystallographic orientation of the grains and the temperature evolution in representative 3D investment cast parts. At present, only the semi-coupled CAFE model was presented and used for regions where the thermal gradient is strong. The solidified path (i.e., the relationship between temperature and volume fraction of solid) is therefore unique and no recalescence can be predicted. The fully coupled 3D CAFE model, in which latent heat released by the mushy cells will be fed back to the FE nodes, is in progress. It will be used as a new tool by industries for the design and understanding of the solidification of investment cast parts.

\section{Acknowledgments}

The authors would like to thank the Commission pour la Technologie et l'Innovation (Bern, CH) (Grant \#2861.1) and the companies ABB Management Ltd (Baden, CH), Calcom S.A. (Lausanne, CH), Howmet Corporation (Whitehall, Michigan, USA), PCC airfoils Inc. (Beachwood, Ohio, USA), Rolls Royce Plc (Derby, UK), SNECMA (Paris, F) and T\&N Technology / AE Turbine Component Ltd (Leeds, UK) for providing the financial support to this research.

\section{References}

1. J.S. Tu and R.K. Foran, J. of Metals 44 (1992) 26.

2. K.O. Yu, J.J. Nichols, and M. Robinson, J. of Metals 44 (1992) 21.

3. D. Goldsmidt, U. Paul, and P.R. Sahm, in Superalloys 1992, Edited by S.D. Antolovich et al., The Minerals, Metals \& Materials Society, pp. 155 (1992).

4. Th. Imwinkelried, J.-L. Desbiolles, Ch.-A. Gandin, M. Rappaz, S. Rossmann, and $\mathrm{Ph}$. Thévoz, in Modeling of Casting, Welding and Advanced Solidification Processes VI, pp. 63, Editcd by T.S. Piwonka ct al., The Mincrals, Mctals \& Materials Socicty (1993).

5. S.L. Cockcroft, M. Rappaz, A. Mitchell, J. Fernihough, and A.J. Schmalz, in Materials for Advanced Power Engineering Part II, pp. 1145, Eds by D. Coutsouradis et al., Netherlands (1994).

6. A. de Bussac and Ch.-A. Gandin, Materials Science and Engineering A, submitted.

7. Ch.-A. Gandin and M. Rappaz, Acta metall. mater. 42 (1994) 2233.

8. M. Rappaz, Ch.-A. Gandin, J.-L. Desbiolles, and Ph. Thévoz, Metall. mater. trans. 27A (1996) 695.

9. Ch.-A. Gandin and M. Rappaz, Acta metall. mater. (1997) in press.

10. Ph. Thévoz, J.-L. Desbiolles, and M. Rappaz, Metall. trans. 20A (1989) 311.

11. W. Kurz, B. Giovanola, and R. Trivedi, Acta metall. 34 (1986) 823.

12. M. Bobadilla, J. Lacaze, and G. Lesoult, J. Cryst. Growth 89 (1988) 531.

13. Ch.-A. Gandin, M. Rappaz, D. West, and B.L. Adams, Metall. mater. trans. 26A (1995) 1543 